\title{
Poroelastic Attributes: A Real-time Determinant Membrane Nanorheology for Receptor Dependent Endocytosis of Targeted Gold Nanoparticles
}

\section{Tanmay Abhay Kulkarni}

Mayo Clinic College of Medicine and Science

\section{Debabrata Mukhopadhyay}

Mayo Clinic College of Medicine and Science

Santanu Bhattacharya ( $\sim$ bhattacharya.santanu@mayo.edu )

Mayo Clinic Florida https://orcid.org/0000-0003-3579-4317

\section{Research}

Keywords: Poroelasticity, Targeted gold nanoparticle, Pancreatic cancer, Receptor mediated endocytosis, Plectin-1 targeting, Drained Poisson's ratio, Effective shear stress, Diffusion coefficient, Pore size and Real-time membrane dynamics

Posted Date: November 15th, 2021

DOI: https://doi.org/10.21203/rs.3.rs-1061474/v1

License: (c) (i) This work is licensed under a Creative Commons Attribution 4.0 International License. Read Full License 


\section{Poroelastic Attributes: A Real-time Determinant Membrane Nanorheology for Receptor Dependent Endocytosis of Targeted Gold Nanoparticles}

Tanmay Kulkarni†, Debabrata Mukhopadhyay $\dagger,+$, Santanu Bhattacharya $\dagger,\}^{*}$

$†$ Department of Biochemistry and Molecular Biology, Mayo College of Medicine and Science, Jacksonville, FL, USA.

$\ddagger$ Department of Physiology and Biomedical Engineering, Mayo College of Medicine and Science, Jacksonville, FL, USA.

*Address Correspondence to: Santanu Bhattacharya, Dept. of Biochemistry and Molecular Biology, Griffin 413, Mayo Clinic Florida, 4500 San Pablo Road S, Jacksonville, FL-32224, Tel: (904) 953-0507; Fax: (904) 953-0277;

E-mail: bhattacharya.santanu@ mayo.edu 


\begin{abstract}
Background: Efficacy of targeted drug delivery using nanoparticles relies on several factors including the uptake mechanisms such as phagocytosis, macropinocytosis, micropinocytosis and receptor mediated endocytosis. These mechanisms have been studied with respect to the alteration in signaling mechanisms, cellular morphology, and linear nanomechanical properties (NMPs). Commonly employed classical contact mechanics models to address cellular NMPs fail to address mesh like structure consisting of bilayer lipids and proteins of cell membrane. To overcome this technical challenge, we employed poroelastic model which accounts for the biphasic nature of cells including their porous behavior exhibiting both solid like (fluid storage) and liquid like (fluid dissipate) behavior
\end{abstract}

Results: In this study, we employed atomic force microscopy to monitor the influence of surface engineering of gold nanoparticles (GNPs) to the alteration of nonlinear NMPs such as drained Poisson's ratio, effective shear stress, diffusion constant and pore dimensions of cell membranes during their uptake. Herein, we used pancreatic cancer (PDAC) cell lines including Panc1, AsPC1 and endothelial cell HUVECs to understand the receptor-dependent and -independent endocytosis of two different GNPs derived using plectin-1 targeting peptide (PTP-GNP) and corresponding scrambled peptide (sPEP-GNP). Compared to untreated cells, in case of receptor dependent endocytosis of PTP-GNPs diffusion coefficient altered $\sim 1264$-fold and $~ 1530$-fold and pore size altered $\sim 320$-fold and $\sim 260$-fold in Panc1 and AsPC-1 cells respectively. Whereas for receptor independent mechanisms, we observed modest alteration in diffusion coefficient and pore size, in these cells compared to untreated cells. Effective shear stress corresponding to $7.38 \pm 0.15$ $\mathrm{kPa}$ and $20.49 \pm 0.39 \mathrm{kPa}$ in PTP-GNP treatment in Panc1 and AsPC-1, respectively was significantly more than that for sPEP-GNP. These results demonstrate that with temporal recruitment of plectin-1 during receptor mediated endocytosis affects the poroelastic attributes of the membrane.

Conclusion: This study confirms that nonlinear NMPs of cell membrane are directly associated with the uptake mechanism of nanoparticles and can provide promising insights of the nature of endocytosis mechanism involved for organ specific drug delivery using nanoparticles. Hence, nanomechanical analysis of cell membrane es using this noninvasive, label-free and live-cell analytical tool can therefore be instrumental to evaluate therapeutic benefit of nanoformulations. 
Keywords- Poroelasticity, Targeted gold nanoparticle, Pancreatic cancer, Receptor mediated endocytosis, Plectin-1 targeting, Drained Poisson's ratio, Effective shear stress, Diffusion coefficient, Pore size and Real-time membrane dynamics 


\section{Background}

Targeted drug delivery using nanoparticle has become a prime focus in cancer treatment to improve its therapeutic outcome [1-3]. Extensive research has been documented to evaluate the efficacy of targeted drug delivery using gold nanoparticles, and identified their uptake mechanisms such as phagocytosis, macropinocytosis, micropinocytosis and receptor mediated endocytosis [47]. These studies have enabled the researchers to achieve significant strides in comprehending alteration in signaling mechanisms, cellular morphology and linear nanomechanical properties (NMPs) that include membrane stiffness, deformation and adhesion using atomic force microscope (AFM) [8-12]. As cell is largely composed of cytosolic fluid and is susceptible to both external and internal biomechanical stimuli, which triggers signaling pathways such as intracellular signal transduction, PI3K/AKT and kinase signalling that corresponds to the cell proliferation, differentiation and apoptosis $[8,9]$. For instance, in cardiovascular system, physical stimuli can be sensed by the cells and in turn transmitted through intracellular signal transduction pathways to the nucleus, thus resulting in cell apoptosis [9]. Fibronectin 1, one of the crucial extracellular matrix (ECM) components affects cellular proliferation and apoptosis of human Glioma cells through PI3K/AKT signaling pathway [13]. Lastly, the role of mechanical stretch in fetal lung cells (E19 type II cells) was studied and the group observed that cellular differentiation occurred via an epidermal growth factor receptor-extracellular regulated protein kinase signaling pathway [14]. On the frontline of nanomechanical studies, cell adhesion mechanism was explored from the force measurements acquired at the multiple-bond level using leukocyte function associated antigen-1 (LFA-1)/intercellular adhesion molecule-1 (ICAM-1) as a model system [15]. Another comprehensive cell membrane stiffness study evaluated in human mammary epithelial cells in various cell cycle as well as microenvironments related to cell-cell contacts led to the findings that these cells become softer as they advance to the tumorigenic phase and then stiffens in their progression to metastatic cells [16]. AFM has also been employed to study the endocytosis in which, the mesh like structure of cellular membrane enables it to interact with the surrounding via exchange of cytosolic fluids as well as allowing uptake of external moieties such as nanoparticles through various endocytosis mechanisms [17, 18]. During this exchange process, cellular cytoskeleton undergoes series of dynamic alterations affecting overall cellular rheology and in turn affecting its NMPs such as membrane stiffness, deformation and adhesion [19-21]. However, alteration in poroelasticity parameters during various endocytosis mechanisms remains to be 
studied and could potentially yield a more in-depth perception that will boost our understanding of endocytosis mechanisms.

Several classical contact mechanics model have been incorporated to study cellular rheology such as Hertz and Sneddon model [22, 23]. However, these models neglect the cellular adhesion with the probe, thus failing to do the desired justice. To address this, in case of larger diameter (micron range) probes, Derjaguin-Muller-Toporov and Johnson-Kendall-Roberts model are commonly incorporated that account for the thermodynamic work of adhesion [24-26]. In addition, power law model is also employed to study viscoelasticity of cells as they tend to be viscous [27-30]. However, these models fall short as they fail to address the biphasic nature of cell's cytoplasm [31]. Poroelasticity model aids in studying the biphasic cytoplasm consisting of porous elastic mesh comprising of cytoskeleton, organelles and macromolecules amidst interstitial cytosolic fluid [32-35]. Poroelasticity model acknowledges that the response of cell membrane to an external stimulus is both time and length scale dependent [36-38]. Poroelasticity model enables quantification of cellular rheology in in terms of drained Poisson's ratio, diffusion coefficient and pore size allowing exploring and correlating them with various biological phenomena [31, 34, 39, 40]. Another study focussed on poroelastic behavior of cells using a micron size bead demonstrating that cytoskeletal components such as actin, microtubules intermediate filaments and myosin affected the diffusion coefficient of cell [31]. In another study, alterations in poroelastic parameters of hepatocellular carcinoma (SMMC-7721) cells treated with fullerenol were explored [39]. Fullerenol treated cells exhibited a significant increase in the pore size and a slight decrease in elastic modulus [39], providing insights into cancer therapies. Thus, poroelastic model which accounts for the biphasic nature of cells including their porous behavior exhibiting both solid like (fluid storage) and liquid like (fluid dissipate) behavior could prove detrimental to our endocytosis understanding.

Present work follows up with our recently published study in which, we explored nano mechanical alterations in pancreatic ductal adenocarcinoma (PDAC) cells during receptor dependent and independent endocytosis mechanisms [41]. We demonstrated correlation between membrane stiffness and surface plectin-1 receptors in PDAC and that with the loss of plectin-1 receptors, PDAC cells became softer when treated with gold nanoparticles conjugated with plectin1 targeted peptide (PTP-GNP). In this work, we explore the dynamic alterations in poroelastic parameters of pancreatic ductal adenocarcinoma cell lines (Panc1 and AsPC-1 cells) when treated 
with PTP-GNP (receptor dependent). We further compare this behavior with human umbilical vein endothelial cells (HUVECs) treated with PTP-GNP as well as scrambled peptide conjugated GNP (sPEP-GNP) and Panc1 and AsPC-1 treated with sPEP-GNP, all exhibiting receptor independent endocytosis mechanisms. Insights into nonlinear poroelastic parameters during receptor dependent and independent endocytosis mechanisms from this study, will aid in understanding cellular behavior during therapeutic targeting.

\section{Results}

\section{AFM tool to evaluate time dependent poroelastic signatures from Force-relaxation curves}

Cells exhibiting poroelastic behavior has been well-established [31, 34, 39, 40]. Several prior studies have indicated severe heterogeneity in cell surface nanomechanics [42, 43]. In addition, irregularities exist in their nanomechanical signatures due to varying cell cycle progression [42]. To overcome these discrepancies, we arrested Panc1, AsPC-1 and HUVEC cells in S-phase and performed ramp scripting on a narrow $\left(500 \times 50 \mathrm{~nm}^{2}\right)$ region over the nuclear membrane as shown in Figure 1A experimental schematic. Arresting cells in particular cell cycle phase yields homogenous cellular population of cells with uniform NMPs [44]. Force-relaxation (F-R) curve is often employed on the cell surface to study the time and length scale alterations in force during the tip-sample interactions [40]. In nanoindentation procedure that yields force separation curve, a typical interaction of the probe with sample surface lasts for a few milliseconds and the probe begins its retraction cycle immediately after attaining the applied triggered force. However, in ramp script procedure to study the time and length scale poroelastic properties, a tip has to remain in contact with the sample surface for a longer duration (typically a few seconds) giving the relaxation in the force once the indentation reaches a predefined value. 

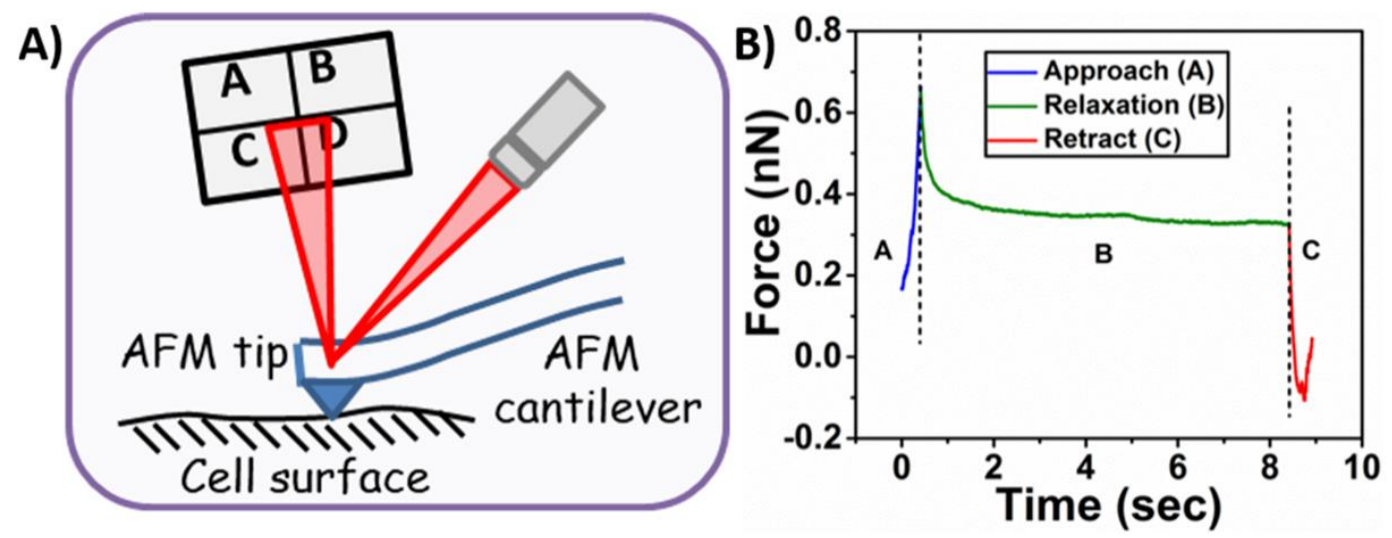

Figure 1. Experimental setup for Force-relaxation (F-R). A) Schematic of AFM tip probing cell surface to acquire an F-R curve. B) A representative F-R curve exhibiting approach, relaxation and retraction steps to constitute an F-R curve.

In this study, we initially performed nanoindentation procedure to determine the force required to achieve a 10\% indentation corresponding to the overall cellular height for Panc1, AsPC-1 and HUVEC. This force was then used in ramp script studies to achieve and maintain indentation of $400 \mathrm{~nm}, 350 \mathrm{~nm}$ and $200 \mathrm{~nm}$ for Panc1, AsPC-1 and HUVECs, respectively. We observed that same force level was able to achieve variable deformation values specific to cell lines and can be attributed to their intrinsic membrane stiffness in the absence of any treatment. A representative F-R curve is shown in Figure 1B. It comprises of three regions viz. approach, relaxation and retraction. During approach segment, the cantilever moves towards the sample at high forward velocity until the desired indentation is achieved. During the approach process, the cytosolic fluid gets trapped inside the cellular membrane. Following which, relaxation in force was monitored over a period of 8 seconds by maintaining the indentation depth. During the relaxation period, force begins to drop exponentially and ultimately reaches a plateau indicating that the redistribution of interstitial fluid and the force imposed by the tip is balanced by the stress in the elastic porous matrix. Final segment of F-R curve is the retract curve during which, the probe overcomes attractive pull-off force exerted by the sample. In order to evaluate poroelasticity parameters, relaxation (middle segment) was analyzed using MATLAB programming software.

\section{Force-Relaxation (F-R) curves}


Intracellular movements of small molecules and large aggregates occur over a broad time scale. Due to which, we only consider the middle segment exhibiting relaxation in force levels at constant indentation over a larger time scale as shown in Figure 2 for various treatments in different cell lines. Here, F-R curve corresponding to PTP-GNP treatment in Panc1 and AsPC-1 cells exhibit receptor dependent endocytosis phenomena as shown in Figure 2A and 2C. However, other treatments such as PTP-GNP and sPEP-GNP in HUVECs as well as sPEP-GNP in Panc1 and AsPC-1 cells represent receptor independent endocytosis mechanisms as shown in Figure 2B and 2D-2F.
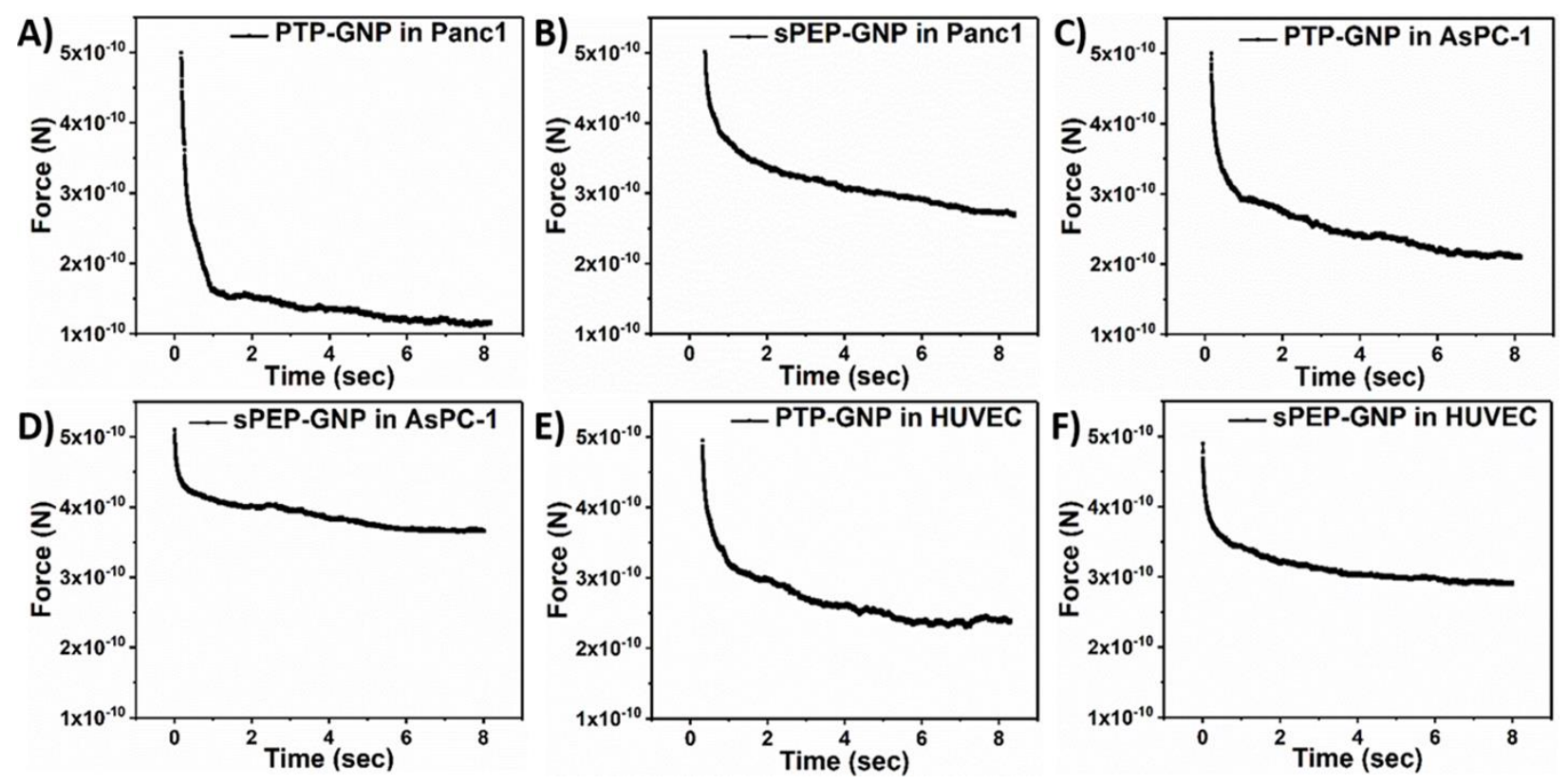

Figure 2. Representative relaxation segments from force-relaxation curves for various GNP formulation treatments in different cell lines. A) PTP-GNP in Panc1. B) sPEP-GNP in Panc1. C)PTP-GNP in AsPC-1. D) PTP-GNP in HUVEC. E) sPEP-GNP in HUVEC. F) sPEP-GNP in AsPC-1.

Before performing F-R curves, nanoindentation experiment was performed on all three cell lines to achieve a force that resulted in less than $10 \%$ deformation. We observed that a force of $500 \mathrm{pN}$ was sufficient to maintain the $10 \%$ criteria that avoided permanent deformations in the cell lines. It is evident that there is a distinct variation in the slope of these curves. F-R curves during receptor dependent endocytosis process presented a steeper slope compared to the receptor independent 
endocytosis scenario. As these curves represent a decayed exponential curve with two distinct slopes, curve fitting is performed on these curves using equation (6) to evaluate characteristic decay time. However, these F-R curves are only qualitative measure of alterations in nonlinear poroelasticity parameters based on slope alterations for respective treatments. Clear distinction in slope between the F-R curves for various treatments and no treatment is evident from Figure 2 and Figure S1 for respective cell lines. To quantify poroelasticity parameters, we used a series of mathematical equations presented above (equations 1-8) and evaluated diffusion coefficient and pore size.

\section{Dynamic alterations in drained Poisson's ratio during receptor dependent and independent endocytosis mechanisms}

As endocytosis is a dynamic process, it becomes crucial to monitor time dependent alterations in cellular nanomechanics. Plectin-1 has been identified as a receptor explicitly overexpressed on pancreatic cancer cells and has been extensively explored as a potential biomarker since its advent [45]. In addition, our recent work exhibits the overexpression of plectin1 in both primary and immortalized pancreatic cancer cell lines including Panc1 and AsPC-1 [41]. Plectin-1 targeted peptide from PTP-GNP binds to the plectin-1 surface receptors in Panc1 cells and internalizes leading to a receptor dependent endocytosis mechanism. Scrambled peptide bound GNP (sPEP-GNP) on the other hand internalizes via receptor independent mechanisms in Panc1 cells as there is no favorable binding complex to the plectin-1 surface receptors. In addition, HUVECs do not possess surface plectin-1 receptors [41], hence the internalization of PTP-GNP and sPEP-GNP occur via receptor independent endocytosis mechanism. To confirm and validate our findings pertaining to alteration in poroelasticity parameters during receptor dependent and independent endocytosis mechanisms, we chose another PDAC cell line, AsPC-1 that is known to exhibit surface plectin-1 receptors [41]. PTP-GNP and sPEP-GNP internalization in AsPC-1 cells occur via receptor dependent and independent endocytosis mechanism, respectively. We first monitored whether the experimental parameters influence the poroelastic parameters in $\mathrm{S}$ phase arrested Panc1 cells over a 20-minute time window, when probed over the same region in the increments of 5-minutes. 

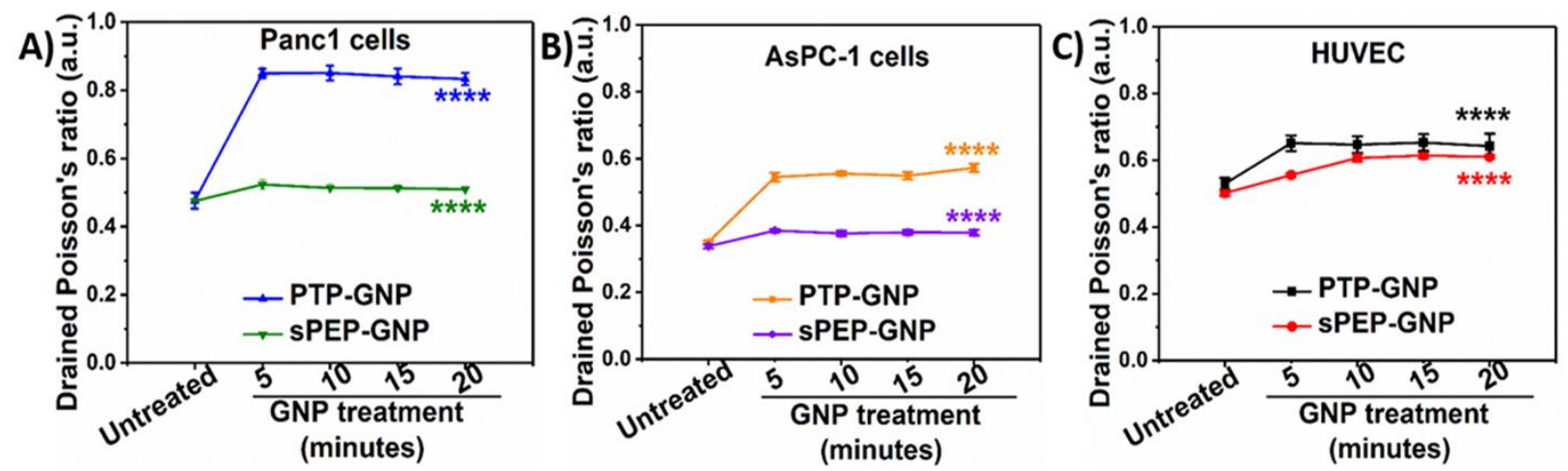

Figure 3. Temporal alteration in drained Poisson's ratio. A) In Panc1 cells during receptor dependent (PTP-GNP in Panc1) and receptor independent (sPEP-GNP in Panc1) endocytosis processes. B) In AsPC-1 cells during receptor dependent (PTP-GNP in AsPC-1) and receptor independent (sPEP-GNP in AsPC-1) endocytosis processes. C) In HUVECs with PTP-GNP and sPEP-GNP treatment both resembling receptor independent endocytosis process. (Statistical significance norm is as follows: $* * * *, p<0.0001$.)

We did not observe any significant alterations in all three poroelastic attributes including drained Poisson's ratio determined from equation (5), diffusion coefficient and pore size as seen from Figure S2a-S2c. In our previous study, we demonstrated that NMPs of cells are significantly governed by the cell phase. As a result, cell synchronization is essential to overcome the heterogeneity in mechanical properties [42]. We verified whether the finding holds true in AsPC1 cells as well by synchronizing the cells in $\mathrm{S}$ phase and comparing their poroelasticity values with asynchronous AsPC-1 cells as shown in Figure S3a-S3c. We observed that while the individual parameters values remain consistent, there is a huge variation in poroelasticity parameters in asynchronous AsPC-1 cells as seen from Figure S3a-S3c. We attribute this inconsistency to the asynchronous nature of AsPC-1 cells. For all the experiments involving AsPC-1 cells, we chose S phase synchronous AsPC-1 cells. And lastly, we evaluated poroelasticity properties of serum starved HUVECs and S phase Panc1 as shown in Figure S4a-S4c. Except for drained Poisson's ratio, other poroelasticity parameters exhibited non-significant difference. Differences in drained Poisson's ratio could be attributed to the intrinsic cellular property. After establishing the baselines for all three cell lines and ensuring that the experimental trigger force does not cause permanent deformations in these cell lines, we monitored dynamic alterations in drained Poisson's ratio of Panc1, AsPC-1 and HUVECs when treated with PTP-GNP and sPEP-GNP over a period of 20 
minutes in the increments of 5 minutes as shown in Figure 3A-3C from equation (5). Untreated Panc1 cells exhibited a drained Poisson's ratio of $0.47 \pm 0.02$ a.u. whereas, untreated AsPC-1 cells displayed lower drained Poisson's ratio (0.34 \pm 0.004 a.u.) compared to the untreated Panc1 cells. Untreated HUVECs exhibited a drained Poisson's ratio of $0.52 \pm 0.01$ a.u. as seen from Figure 3A3C. Upon PTP-GNP treatment in Panc1 cells and AsPC-1 cells, we observed an immediate increase of 1.8-fold and 1.6-fold in drained Poisson's ratio in the initial 5 minutes (drained Poisson's ratio of $0.85 \pm 0.02$ a.u. in Panc1 and $0.54 \pm 0.01$ a.u. in AsPC-1 cells, respectively), thereafter remaining more or less constant over the 20-minute time window as seen from Figure 3A and 3B. Although we observed an increase in drained Poisson's ratio for receptor independent endocytosis, peak increase in drained Poisson's ratio was observed at various time points for various cells with treatments. For instance, drained Poisson's ratio for PTP-GNP in HUVEC (drained Poisson's ratio of $0.65 \pm 0.02$ a.u.) and sPEP-GNP in Panc1 (drained Poisson's ratio of $0.52 \pm 0.01$ a.u.) both reached a peak at 5-minute timepoint, thereafter remaining more or less constant over the 20 minute time window as shown in Figure 3A and 3C. Interestingly, in the case of sPEP-GNP treatment in HUVEC, peak increase in drained Poisson's ratio was observed at 15minute time point (drained Poisson's ratio of $0.61 \pm 0.008$ a.u.), thereafter remaining constant for the rest of the 20-minute time window. Similarly, we observed that when AsPC-1 cells were treated with SPEP-GNP, the treatment caused the drained Poisson's ratio to increase to $0.38 \pm 0.005$ a.u. at the 5-minute time point and remain consistent for the remaining duration as seen from Figure 3B. Such behavior in receptor dependent and independent endocytosis mechanism in AsPC-1 cell line was consistent with Figure 3A and 3C. Although an increase in drained Poisson's ratio was observed for both receptor dependent and independent scenarios, fold change increase in receptor dependent endocytosis case was substantial compared to receptor independent as evident from Figure 3A-3C.

\section{Dynamic alterations in shear stress during receptor dependent and independent endocytosis mechanisms}

The AFM tip rapidly indenting into the cell membrane surface, exerts a force perpendicular to the cell membrane. As a result, a shear force component arises parallel to the cellular crosssection [46]. Moreover, shear stress is correlated to the cell membrane stiffness as shown in equation 2. Previously, we have shown a contrary alteration in membrane stiffness during receptor 
dependent and independent endocytosis mechanisms [41]. Here, we monitored the alterations in shear stress during these endocytosis mechanisms and further evaluate the effective change in the shear stress between the drained and undrained scenario from equations 3 and 4 .
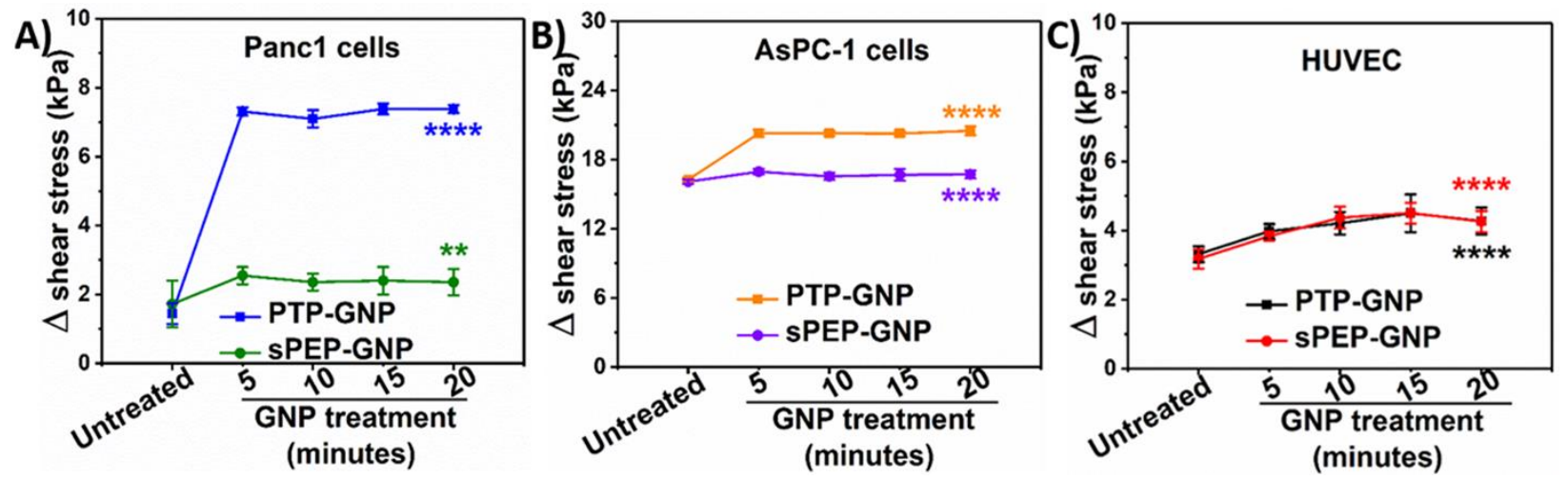

Figure 4. Temporal alteration in effective change in shear stress during the transition from undrained to drained state. A) In Panc1 cells during receptor dependent (PTP-GNP in Panc1) and receptor independent (sPEP-GNP in Panc1) endocytosis processes. B) In AsPC-1 cells during receptor dependent (PTP-GNP in AsPC-1) and receptor independent (sPEP-GNP in AsPC-1) endocytosis processes. C) In HUVECs with PTP-GNP and sPEP-GNP treatment both resembling receptor independent endocytosis process. (Statistical significance norm is as follows: **, p<0.01; $* * * *, \mathrm{p}<0.0001$.)

In the undrained case, shear stress is dictated by indentation, which varies for cell lines. The applied force and the tip radius are constant parameters. We observed that AsPC-1 cell lines exhibited maximum shear stress during the undrained state with $24.1 \mathrm{kPa}$, whereas, HUVEC and Panc1 cell lines exhibited $10.4 \mathrm{kPa}$ and $8.52 \mathrm{kPa}$ shear stress, respectively determined from equation (3). After the built-up interstitial pressure during rapid indentation was dissipated (drained state), we observed that the shear stress was significantly lower than the undrained case. Different cell lines in untreated state exhibited varying drained shear stress such as 7.167 \pm 0.09 $\mathrm{kPa}, 6.94 \pm 0.19 \mathrm{kPa}$ and 7.94 $\pm 0.12 \mathrm{kPa}$ for HUVECs, Panc1 and AsPC-1 cell lines, respectively as seen from Figure S4a-S4c. For receptor dependent scenario (PTP-GNP in Panc1 and AsPC-1), we observed an immediate decrease in drained shear stress at 5-minute time point with values corresponding to $1.21 \pm 0.11 \mathrm{kPa}$ and $3.83 \pm 0.3 \mathrm{kPa}$, respectively compared to the untreated cells as 
shown in Figure S5a and S5b. For PTP-GNP treatment in Panc1 and AsPC-1 cell lines, maximum drop in drained shear stress value was observed at 15-minute time point and 20-minute time point with value corresponding to $1.13 \pm 0.15 \mathrm{kPa}$ and $3.61 \pm 0.38 \mathrm{kPa}$, respectively as evaluated from equation (4). Furthermore, for receptor independent scenario (PTP-GNP and sPEP-GNP in HUVECs, sPEP-GNP in Panc1 and AsPC-1), we observed a significantly lesser drop in drained shear stress compared to the receptor dependent case as seen from Figure S5a-S5c. In the case of HUVECs, both GNP treatments induced a decreasing trend in drained shear stress, which was maximum at 15-minute time point. With values corresponding to $5.91 \pm 0.54 \mathrm{kPa}$ and $5.91 \pm 0.3 \mathrm{kPa}$ for PTP-GNP and sPEP-GNP treatments, respectively as seen from Figure S5c. For sPEP-GNP treatments in Panc1 and AsPC-1 cell lines, we observed a maximum decrease in drained shear stress corresponding to 15-minute time point (Panc1 cells) and 5-minute time point (AsPC-1 cells) with values corresponding to $5.9 \pm 0.25 \mathrm{kPa}$ and $7.15 \pm 0.24 \mathrm{kPa}$, respectively as seen from Figure S5a and S5b. In terms of effective change in shear stress transitioning from undrained to drained scenario for various cell lines, we observed that receptor dependent case exhibited maximum alteration with values corresponding to $7.38 \pm 0.15 \mathrm{kPa}$ and $20.49 \pm 0.39 \mathrm{kPa}$ for PTP-GNP treatment in Panc1 and AsPC-1 cell lines at 15-minute and 20-minute time point, respectively as seen from Figure 4A and 4B. In the case of receptor independent endocytosis mechanism, we observed an effective change in shear stress for Panc1 and AsPC-1 cell lines treated with sPEP-GNP with values corresponding to $2.4 \pm 0.4 \mathrm{kPa}$ and $16.9 \pm 0.24 \mathrm{kPa}$ at 15 -minute and 5-minute time point, respectively (Figure 4A and 4B). For HUVECs treated with both PTP-GNP and sPEP-GNP (both resembling receptor independent endocytosis mechanisms), we observed an effective change in shear stress at 15 -minute time point with values corresponding to $4.5 \pm 0.5 \mathrm{kPa}$ and $4.5 \pm 0.3 \mathrm{kPa}$, respectively as seen from Figure 4C. Therefore, from these observations we conclude that the effective change in shear stress for receptor dependent endocytosis scenario was significantly more prominent than the receptor independent scenario for respective cell lines.

\section{Alterations in diffusion coefficient signature during receptor dependent and independent endocytosis mechanisms}

We then monitored the diffusion coefficient for both receptor dependent and receptor independent endocytosis mechanisms in Panc1, HUVECs and AsPC-1 cell lines as shown in Figure 5A-5C. Diffusion coefficient is a measure of the diffusion of cytosolic fluid that is trapped 
inside the cell membrane due to the rapid pressure buildup during approach phase of F-R experiment. During the relaxation segment, this built up pressure is released in the form of the cytosolic fluid diffusion causing a change in diffusion coefficient, which was evaluated using equation (7). Untreated Panc1 cells and AsPC-1 cells exhibited a diffusion coefficient of 1.58x10${ }^{15} \pm 2 \times 10^{-16} \mathrm{~m}^{2} / \mathrm{s}$ and $7.51 \times 10^{-16} \pm 4.3 \times 10^{-18} \mathrm{~m}^{2} / \mathrm{s}$, respectively, whereas HUVECs exhibited a diffusion coefficient of $1.59 \times 10^{-15} \pm 9.96 \times 10^{-17} \mathrm{~m}^{2} / \mathrm{s}$ as seen from Figure $5 \mathrm{~A}-5 \mathrm{C}$. We observed that during the receptor dependent endocytosis mechanism (PTP-GNP in Panc1 and AsPC-1 cells), there occurred $\sim 1264$-fold change and $\sim 1530$-fold change in diffusion coefficient (2x10${ }^{12} \pm 3.22 \times 10^{-14} \mathrm{~m}^{2} / \mathrm{s}$ in Panc1 and $1.15 \times 10^{-12} \pm 2.28 \times 10^{-14} \mathrm{~m}^{2} / \mathrm{s}$ in AsPC-1 cells) after the 5minutetime point and 20-minute time point for Panc1 and AsPC-1 cells, respectively as seen from Figure 5A and 5B. Upon PTP-GNP treatment in AsPC-1 cells, we observed a systematic increase in the diffusion coefficient upto 20-minute time window. Although we observed an increasing trend in diffusion coefficient, we maintained the 20-minute time window for consistency. On the other hand, for receptor independent endocytosis mechanisms, a very minimal increase in diffusion coefficient ( 1.1-fold) was observed for PTP-GNP in HUVEC $\left(1.74 \times 10^{-15} \pm 7.4 \times 10^{-17} \mathrm{~m}^{2} / \mathrm{s}\right)$ and sPEP-GNP in HUVEC $\left(1.75 \times 10^{-15} \pm 4.84 \times 10^{-17} \mathrm{~m}^{2} / \mathrm{s}\right)$ compared to the respective controls, as shown in Figure 5C. No significant change in diffusion coefficient for sPEP-GNP treatment in Panc1 was observed during the 5-minute time point. Thereafter, we observed a systematic increase for the next two time points for receptor independent endocytosis process. A fold-increase of 1.61, 1.38 and 1.27 was observed in the case of PTP-GNP treatment in HUVEC, sPEP-GNP in HUVEC and sPEP-GNP in Panc1, respectively as seen from Figure 5A and 5C. For receptor independent endocytosis, we observed non-significant changes in diffusion coefficient post 5-minute time point until 20 minutes. 


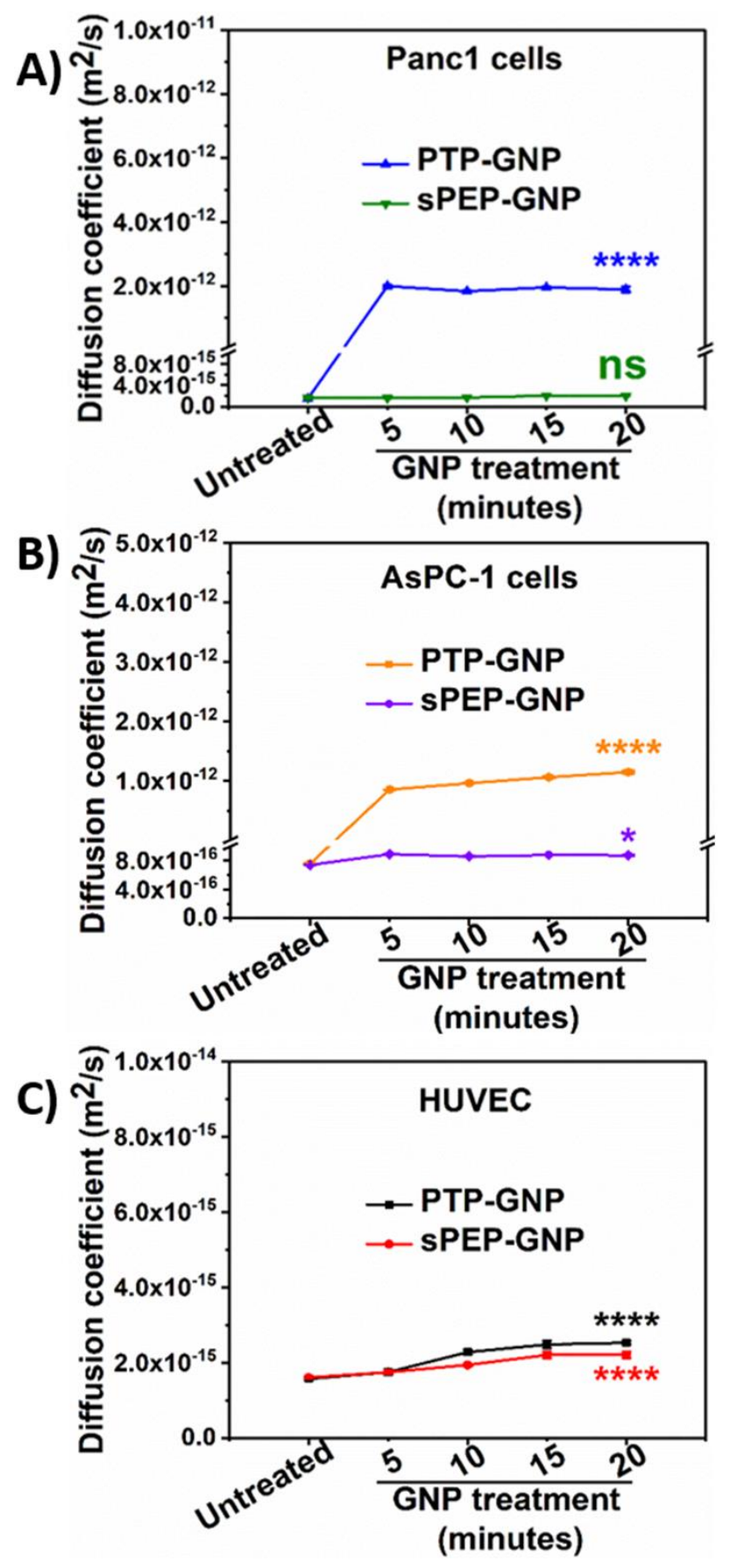

Figure 5. Temporal alteration in diffusion coefficient. A) In Panc1 cells during receptor dependent (PTP-GNP in Panc1) and receptor independent (sPEP-GNP in Panc1) endocytosis processes. B) In AsPC-1 cells during receptor dependent (PTP-GNP in AsPC-1) and receptor independent (sPEP-GNP in AsPC-1) endocytosis processes. C) In HUVECs with PTP-GNP and sPEP-GNP treatment both resembling receptor independent endocytosis process. (Statistical significance norm is as follows: $n s$, not significant; *,p<0.05; ****, $<<0.0001$.) 
We further observed the behavior of sPEP-GNP in AsPC-1 cells also resembling receptor independent endocytosis over the same time window as shown in Figure 5B. When AsPC-1 cells were treated with sPEP-GNP, we observed an increase in the diffusion coefficient after 5 minutes of the treatment with value corresponding to $8.92 \times 10^{-16} \pm 2.03 \times 10^{-18} \mathrm{~m}^{2} / \mathrm{s}$, thereafter remaining constant over a 20-minute time window. Such a behavior was consistent in receptor independent endocytosis mechanisms and associated change in diffusion coefficient in Panc1 and HUVECs with various treatments as seen from Figure $5 \mathrm{~A}-5 \mathrm{C}$. These results indicate the true dynamic nature of the endocytosis mechanism and diffusion coefficient could serve as an important characteristic to differentiate these endocytosis mechanisms.

\section{Alterations in pore size signature during receptor dependent and independent endocytosis mechanisms}

We further probed into the alterations associated with the pore size factor with both receptor dependent and independent endocytosis mechanisms, that were evaluated using equation (8). Consistent with the observed trends in the case of drained Poisson's ratio and diffusion coefficient, pore size too altered drastically for receptor dependent endocytosis mechanism as shown in Figure 6A and 6B. Untreated Panc1, AsPC-1 and HUVECs possessed a pore size of $1.76 \times 10^{-20} \pm 3.02 \times 10^{-}$ ${ }^{22} \mathrm{~m}^{2} 1.65 \times 10^{-20} \pm 3.1 \times 10^{-22} \mathrm{~m}^{2}$ and $1.41 \times 10^{-20} \pm 1.17 \times 10^{-22} \mathrm{~m}^{2}$, respectively as seen from Figure $6 \mathrm{~A}-$ 6C. A fold increase of $\sim 320\left(5.69 \times 10^{-18} \pm 9.29 \times 10^{-20} \mathrm{~m}^{2}\right)$ was observed during the initial 5-minute time window for PTP-GNP treatment in Panc1 as seen from Figure 6A. Thereafter, a sharp drop in pore size was observed, ( 100-fold decrease compared to the $5^{\text {th }}$ minute time point $)$ following which we observed a systematic increase as seen from Figure 6A. This inconsistent trend for pore size in receptor dependent endocytosis mechanism could be attributed to the dynamic endocytosis mechanism during which, surface plectin-1 receptor expression levels are constantly changing due to the internalization leading to pore size variability. At the $20^{\text {th }}$ minute time point, it regained its fold increase to that of $5^{\text {th }}$ minute time point. When AsPC-1 cells were treated with PTP-GNP, we observed $\sim 250$-fold increase in pore size at the end of 5 minutes $\left(4.25 \times 10^{-18} \pm 3.88 \times 10^{-20} \mathrm{~m}^{2}\right)$ compared to untreated AsPC-1 cells as seen from Figure 6B. Here, we observed a systematic increasing trend in pore size until the 20 -minute time window, where peak pore size of $5.13 \times 10^{-}$ ${ }^{18} \pm 2.65 \times 10^{-20} \mathrm{~m}^{2}$ was observed, which was different from PTP-GNP treatment in Panc1 cells. 

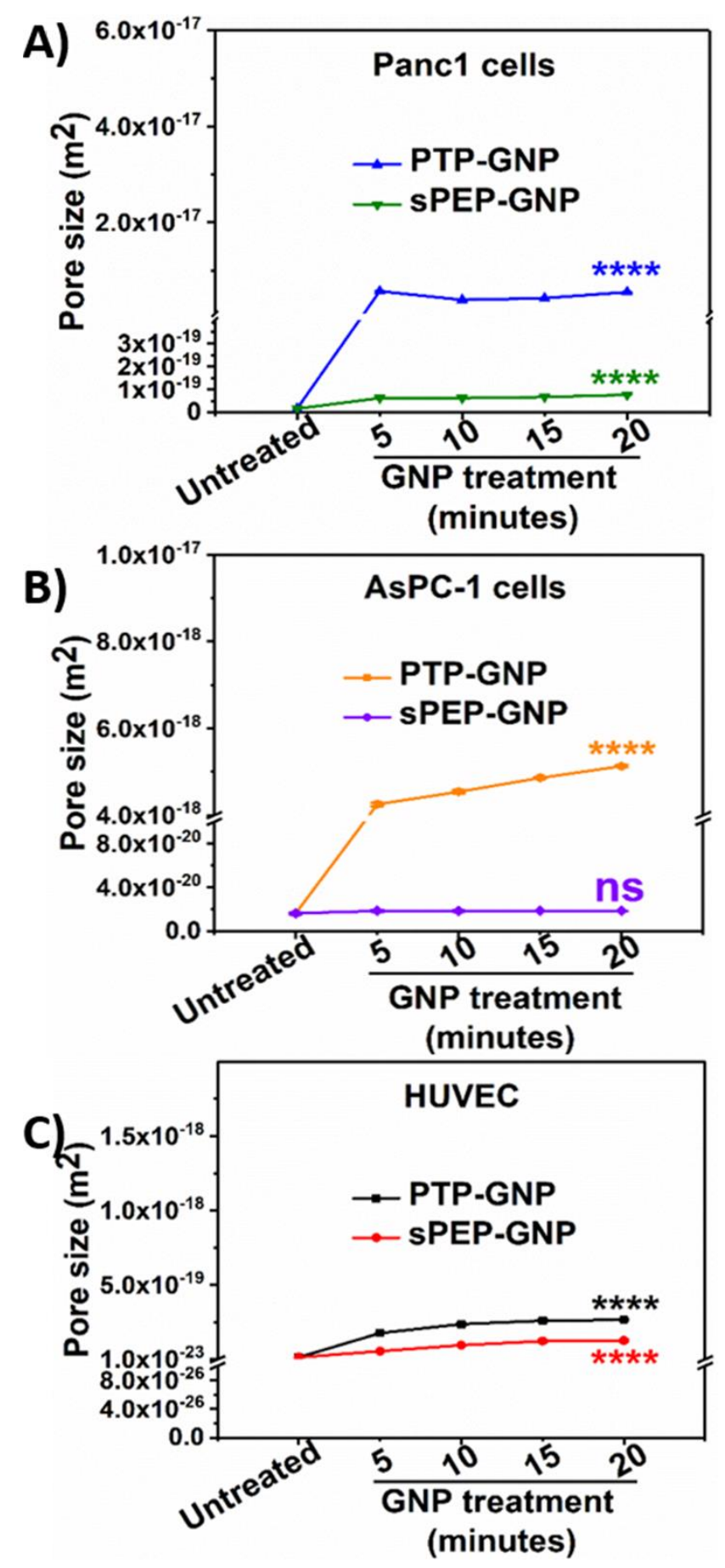

Figure 6. Temporal alteration in pore size. A) In Panc1 cells during receptor dependent (PTPGNP in Panc1) and receptor independent (sPEP-GNP in Panc1) endocytosis processes. B) In AsPC-1 cells during receptor dependent (PTP-GNP in AsPC-1) and receptor independent (sPEPGNP in AsPC-1) endocytosis processes. C) In HUVECs with PTP-GNP and sPEP-GNP treatment both resembling receptor independent endocytosis process. (Statistical significance norm is as follows: ns, not significant; ****, p<0.0001.) 
Within receptor independent scenarios, the rate of increase in pore size differed as evident from the slope changes. PTP-GNP in HUVECs showed maximum fold change at the end of $20^{\text {th }}$ minute time window $\left(2.65 \times 10^{-19} \pm 5.94 \times 10^{-21} \mathrm{~m}^{2}\right)$ exhibiting $\sim 18$-fold increase compared untreated HUVECs, whereas sPEP-GNP in HUVEC exhibited a pore size of $1.26 \times 10^{-19} \pm 7.56 \times 10^{-21} \mathrm{~m}^{2}$ ( 9.8-fold increase) and sPEP-GNP in panc1 displayed a pore size of $7.75 \times 10^{-20} \pm 5.04 \times 10^{-22} \mathrm{~m}^{2}$ ( 4.4-fold increase) as shown in Figure 6A and 6C. Similar to the other poroelastic parameters mentioned before, we confirmed the changes in pore size during receptor independent endocytosis mechanisms using AsPC-1 cell line and maintained consistency in experimental parameters and time window. . The receptor independent endocytosis mechanism scenario exhibited by the sPEPGNP treated AsPC-1 cells did not show a significant increase in the pore size over the 20-minute time window. The maximum increase was observed at $5^{\text {th }}$ minute, corresponding to the pore size value of $1.88 \times 10^{-20} \pm 1.28 \times 10^{-22} \mathrm{~m}^{2}$ as seen from Figure $6 \mathrm{~B}$. These results confirm the dynamic nature of the endocytosis mechanisms and the alterations in the cellular rheology introduced by the endocytosis. The pore size can be further explored to form one of the key signatures along with diffusion coefficient to distinguish various endocytosis mechanisms.

\section{Discussion}

Several studies have reported that the components of cellular cytoskeleton such as actin filaments, microtubules, intermediate keratin filaments and myosin play a pivotal role in regulating cellular NMPs [20, 47-50]. However, these studies focused on liner nanomechanical characteristics such as membrane stiffness, adhesion and deformation. Typical soft samples such as cells possess sponge-like porous elastic matrix through which exchange of interstitial fluid as well as nanoparticles takes place via various endocytosis mechanisms [51, 52]. Depending on the endocytosis mechanisms, the cell membrane undergoes dynamic alterations, which in turn affect their mechanical properties [41]. Also, as endocytosis process is dynamic in nature, it becomes vital to study the temporal dynamicity in nanomechanical alterations at the cell membrane [41, 53]. Intracellular exchange of small molecules and large aggregates occur over a broad time scales $[16,54]$. A conventional nanoindentation experiment that typically occurs over a few millisecond periods yields instantaneous linear NMPs. Whereas force-relaxation (F-R) measurements shed further insights into cellular nanomechanics in terms of poroelasticity parameters better suited for such broad time scale processes. In the field of AFM, it is immensely vital to maintain the 
deformation within $10 \%$ of the sample height to prohibit excess prodding that might result into permanent deformation in soft samples such as cells. In our study, we have optimized the applied force such that the overall deformation falls within the above-mentioned criteria.

Thus far, several studies have focused on the morphological alteration in cell membrane such as the pit formation and membrane ruffling during nanoparticle uptake process [45, 55]. Some prior studies including ours, focused on time-dependent alterations in membrane stiffness during receptor mediated endocytosis process $[41,56]$. And while the biochemics involved in receptor dependent endocytosis process is well understood, alterations in poroelastic parameters during receptor dependent and independent endocytosis mechanisms have not been studied before. Here, using the AFM tool we study the dynamic alterations in poroelastic properties of cell membrane in Panc1 and AsPC-1 cells treated with PTP-GNP (receptor dependent) as well as Panc1 and AsPC-1 cells treated with sPEP-GNP, HUVECs treated with PTP- and sPEP-GNP (receptor independent) over a time window of 20 minutes, which was sufficient to capture the dynamic events as significant changes occurred during the initial 5 minutes time point and remained more or less constant at the end of 20-minute time window. Plectin-1 protein, a novel biomarker for pancreatic cancer detection provides and maintains cellular mechanical integrity [45, 57]. During receptor dependent endocytosis process, PTP-GNP binds to the membrane surface plectin-1 receptors and internalize, due to which there was a significant decrease in membrane plectin-1 expression as seen from the schematic Figure 7A. Due to the absence of surface plectin-1 receptors in normal endothelial cells such as HUVECs, receptor independent mechanisms are more prevalent as shown in schematic Figure 7A. With the loss in plectin-1, Panc1 cells appear significantly softer with time [41]. Herein, we attribute that the loss of mechanical integrity leads to opening/widening of pores that can allow the built-up interstitial pressure (due to rapid indentation) to dissipate rapidly thus, increasing diffusion coefficient and pore during receptor dependent mechanism. 


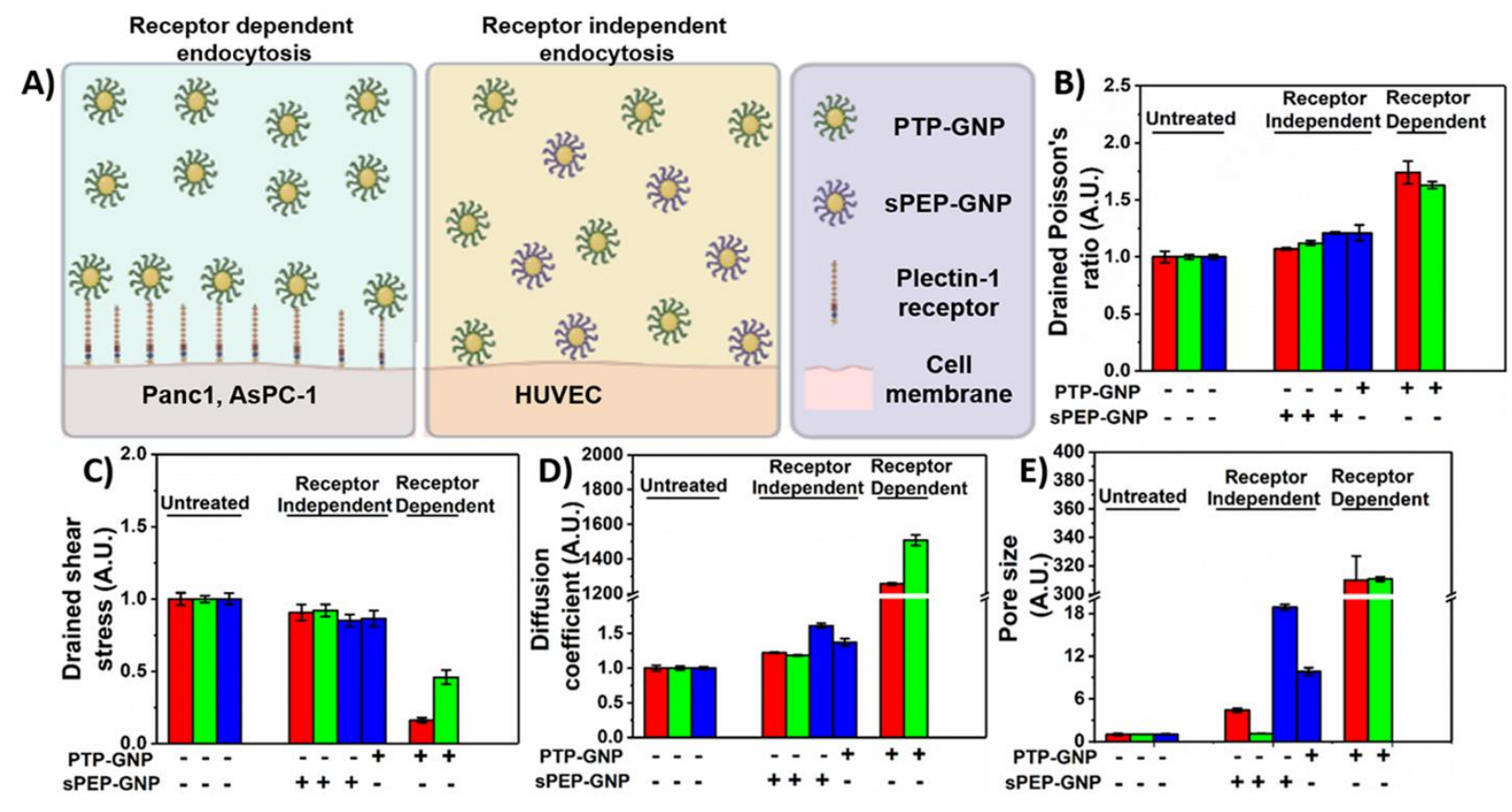

Figure 7. Comparative analysis of poroelasticity parameters influenced by receptor dependent and independent endocytosis processes. A) Schematic of receptor dependent and receptor independent endocytosis process. Normalized poroelasticity parameters at the end of 20minute time window corresponding to B) Drained shear stress. C) Drained Poisson's ratio. D) Diffusion coefficient. E) Pore size.

During receptor independent endocytosis mechanism, the uptake of these GNPs might create significantly smaller pores through which, the built-up pressure is released. As the plectin-1 receptors, which maintain cellular mechanical integrity are not involved in this mechanism, we observed diffusion coefficient and the pore size to be significantly lesser compared to the receptor dependent endocytosis mechanism. We observed multifold increase in poroelastic parameters during receptor dependent endocytosis mechanism compared to receptor independent endocytosis as shown in Figure 7B-7E and attribute such a behavior to the presence of surface plectin-1 receptors, which has been shown to be present in Panc1 and AsPC-1 cells [41].

With the advent of technology, using AFM we can now evaluate nonlinear (poroelastic) properties such as drained Poisson's ratio, diffusion coefficient and pore size of soft biological samples [58-63] in addition to the linear NMPs and serve several advantages over conventional techniques such as optical tweezers, magnetic tweezing cytometry and mechanical micropipette 
aspiration [54]. AFM's unique capability of applying an external trigger force to study the response of the sample in a physiologically favorable condition at nanometer spatial resolution makes it a popular choice. As the indenter is rapidly approached and indented on to the cell membrane, an interstitial fluid pressure builds up within the cell giving rise to undrained condition. The redistribution of the interstitial fluid pressure (drained condition) is both time and length scale dependent and through force-relaxation, the effect of osmotic and cytoskeletal perturbations on cellular rheology can be understood with poroelasticity model [34]. Here, we observed a significant change in effective shear stress as the cell transitions from undrained to drained condition. It is essential to study the effect of external stimulus on cells as they are often subjected to fluid shear stress generated by the blood flow in the vascular microenvironment and interstitial flows in the tumor microenvironment [64]. In one of the studies using biomimetic microfluidic system, it was shown that the uptake of polystyrene nanoparticles in biomimetic dynamic conditions (cells are under higher shear stress than static system) by cancer cells was higher than that in a static system [64]. Similar study has been performed in different cell lines such as Human Embryonic Kidney (HEK) 293T cells, Panc1 cells, human lung adenocarcinoma (A549) cells and human colorectal adenocarcinoma (HT29) cells. It was observed that in the presence of biomimetic shear stress, the cellular uptake of Doxorubicin was significantly higher than in static environment, which also affected its cell killing efficiency [65]. In our study, we observed a significant increase in effective change in shear stress when the cell transitions from undrained to drained state in the case of receptor mediated endocytosis mechanism. Our study supports the results mentioned in other studies and quantifies realtime alterations in effective shear stress during dynamic endocytosis process. Lastly, alterations in linear as well as nonlinear NMPs in biological cells rely heavily on actin filaments, microtubules as well as keratin intermediate filaments (KIF) [66]. Previously, a direct correlation between the structure of the KIF network and its local mechanical properties in alveolar epithelial cells was demonstrated [62]. In that study, shear stress applied across the cellular surface induced structural remodeling of the KIF due to mediation of the phosphorylation of K18pSer33, an essential protein involved in reorganization of the KIF network. Leading to increased cell membrane stiffness [66]. Our observations from the present and previously published study indicate a direct correlation between cell membrane stiffness and drained shear stress in receptor dependent endocytosis process [41]. 
We observed significant increase in diffusion coefficient and pore size during the receptor mediated endocytosis mechanism. Although, we observed a small increase in the diffusion coefficient and pore size during receptor independent mechanisms, they were approximately 1000fold and 200-fold lesser, respectively than corresponding values observed during receptor dependent mechanisms as shown in Figure 7D and 7E. While we observe multi-fold increase in diffusion coefficient, drained Poisson's ratio and pore size; drained shear stress was observed to be following a decreasing trend contrary to the aforementioned poroelastic parameters. The folddrop drained in shear stress in receptor dependent endocytosis was significantly more than receptor independent endocytosis as seen from Figure 7C. Moreover, significant alterations in their values occurred during the initial 5-minute time window after respective GNP-formulation treatment. From our previous study, we demonstrated a positive correlation between membrane stiffness and surface plectin-1 expression levels in Panc1 cells [41]. However, these fold changes in the membrane stiffness were several decades-fold lesser than the diffusion coefficient and pore size as observed in this study. Thus, these nonlinear poroelastic parameters prove to be more definitive indicators of the dynamic alterations in membrane dynamics.

Similar mechanism has been reported in HeLa S3 cells [34]. In this study, cytoskeletal component such as actin filaments and not the microtubules and keratin intermediate filaments played a key role in regulating cellular rheology [34]. In fact, the diffusion coefficient strongly depended on the actomyosin [34]. Moreover, depolymerizing the F-actin cytoskeleton decreased membrane stiffness, increased pore size and resulted in an overall increase in diffusion coefficient, a behavior similar to our observations [34, 41]. Similarly, in MDA-MB-231 cells, it was observed that with higher indenting velocities as the cells appeared stiffer, they were observed to be less poroelastic with lower diffusion coefficient and reduced pore size [40] coinciding with our results. And finally, poroelastic parameters of hepatocellular carcinoma (SMMC-7721) cells when subjected to fullerenol treatment were observed to be higher (both diffusion coefficient and pore size) with a decrease in cell membrane stiffness corroborating the relationship between membrane stiffness, diffusion coefficient and pore size similar to our observation [39, 41]. Above studies aid us in establishing a correlation in poroelastic parameters and cell membrane stiffness. To the best of our knowledge, our study is first of its kind to shed light into the nonlinear poroelastic alterations in membrane dynamics during receptor mediated endocytosis process. This will not only serve as key signatures to distinguish receptor dependent from independent endocytosis mechanism but 
will also aid us in improving our understanding of cellular rheology changes during various endocytosis mechanisms.

\section{Conclusion}

Employing AFM's ramp script technique that yielded F-R curves, poroelastic alterations in membrane dynamics of Panc1, AsPC-1 and HUVECs were evaluated. Permutation and combination of PTP-GNP and sPEP-GNP treatments in above cell lines gave rise to the receptor dependent and independent endocytosis mechanisms. The loss of plectin-1 receptor proteins at Panc1 and AsPC-1 cell membrane during receptor mediated endocytosis process opened/widened the pores that allowed the built-up interstitial pressure during rapid indentation to be released exhibiting a higher diffusion coefficient and pore size compared to receptor independent endocytosis mechanism. Poroelastic parameters proved to be more definitive indicators of the dynamic alterations in membrane dynamics compared to linear parameters during various endocytosis processes. We demonstrate that AFM is a promising tool to segregate receptor dependent from independent process based on poroelastic parameters.

\section{Experimental Section}

\section{Cell culture}

Human pancreatic cancer cell line Panc1 and AsPC-1 were purchased from American Type Culture Collection (ATCC) and used with no further validations. Panc1 and AsPC-1 cells were cultured at $\sim 60-70 \%$ confluency in separate $60 \mathrm{~mm}$ dishes with Gibco Dulbecco's Modified Eagle media (DMEM); supplemented with 10\% Fetal Bovine Serum (FBS) and 1\% Penicillin Streptomycin at $37^{\circ} \mathrm{C}$ in a humidified $5 \% \mathrm{CO}_{2}$ atmosphere. These cells were then arrested in $\mathrm{S}$ phase using $20 \mu \mathrm{g} / \mathrm{mL}$ aphidicolin for 12 hours according to our previous study [42]. Prior to the experiments, culture media was replaced with Leibovitz-15 (L-15) media containing aphidicolin to continue arresting cells in $\mathrm{S}$ phase during the course of the experiments. Human umbilical vein endothelial cells (HUVECs) purchased from Lonza, were cultured at 60-70\% confluency in a 60 $\mathrm{mm}$ dish with on plates coated with $30 \mathrm{mg} / \mathrm{mL}$ collagen type 1 and cultured in endothelial basal medium (EBM) supplemented with EBM-MV Bullet Kit (5\% fetal bovine serum in EC basic medium with $12 \mu \mathrm{g} / \mathrm{mL}$ bovine brain extract, $1 \mu \mathrm{g} / \mathrm{mL}$ hydrocortisone, $1 \mu \mathrm{g} / \mathrm{mL}$ GA1000). HUVECs were serum starved $(0.25 \%$ fetal bovine serum $)$ for 6 hours prior to the experiment [67]. Just before the experiment, EBM was replaced with L-15 media with 2\% FBS to 
continue to arrest HUVECs. Both Panc1 and HUVECs were arrested to overcome the heterogeneity in their NMPs arising from cells in various phases.

\section{Synthesis of gold nanoparticles}

Synthesis of gold nanoparticles conjugated with plectin-1 targeted peptide (PTP-) or scrambled peptide (sPEP-) has been described in our previous studies (published in Nanoscale) [41, 68]. Briefly, Gold (III) chloride trihydrate and peptide were dissolved in Milli-Q water in 10:1 molar ratio under continuous stirring for $2 \mathrm{~min}$ at $37^{\circ} \mathrm{C}$. Then, $1 \mathrm{M} \mathrm{NaOH}$ was added drop by drop until the overall $\mathrm{pH}$ of the solution was achieved at 12 and further subjected to continuous stirring for 16 hours. GNPs were isolated by ultracentrifugation at 38K RPM using Beckmann Optima L80 XP ultracentrifuge (purchased from Beckman Coulter Inc.). Supernatant was removed carefully to yield pellet of PTP-GNP or sPEP-GNP sedimented at the bottom of the centrifuge tube. Finally, Milli-Q water was added to the collected pellet to make the final volume same as the reaction

mixture. For experiment purpose, above solutions were further diluted 10-fold to constitute a working stock solution and stored at $4^{\circ} \mathrm{C}$ until further use. A comprehensive characterization of these gold nanoparticle formulations have been performed in our previous study [68], where the overall size of PTP-GNP and sPEP-GNP was $\sim 5 \mathrm{~nm}$ and the addition of external surface moieties did not significantly alter their dimensions.

\section{Atomic force microscopy}

Dimension Fast Scan with Scanasyst (Bruker Corp.) AFM was employed with LC CAL A probes to perform ramp script analysis mode that yielded force-relaxation (F-R) curves on both Panc1 and HUVECs in fluid environment to preserve their mechanical integrity. $60 \mathrm{~mm}$ culture dishes consisting of Panc1 or HUVECs were held on to the AFM stage using a custom-built plate holder. Due to the overall heterogeneity in cell membrane, we chose a 500 x $50 \mathrm{~nm}^{2}$ region over the nucleus for all the AFM studies [42, 69]. Bruker's MIRO View allowed us to maintain the location of these data points to be constant throughout the dynamic studies over a period of 20 minutes. This spherical probe with nominal spring constant of $0.1 \mathrm{~N} / \mathrm{m}$ and tip radius of $70 \mathrm{~nm}$ was calibrated in Milli-Q water to determine the spring constant and deflection sensitivity of 0.06 $\mathrm{N} / \mathrm{m}$ and $55.7 \mathrm{~nm} / \mathrm{V}$, respectively. Then we optimized the Quantitative Nanomechanical Mapping (QNM) sync distance for the probe at $1 \mathrm{KHz}$ frequency by measuring its deflection sensitivity to 
be within $\pm 5 \%$ range of the tip's deflection sensitivity measured via thermal calibration for accurate tip-sample interaction. Experimental approach adopted to study the poroelasticity parameters was force-relaxation (F-R). F-R allows monitoring the relaxation in the force at a constant height (indentation). Our sample set comprised of 7 cells: each cell with 7 data points over a 20-minute time window in the increments of 5 minutes. All the experiments were performed at $37^{\circ} \mathrm{C}$ maintained using a temperature-controlled AFM stage.

\section{Poroelasticity model theory}

The detailed explanation of the theoretical model of poroelasticity and its application is well described in the previous studies [70,71]. Poroelasticity model yields quantitative parameters such as the drained Poisson's ratio $\left(U_{d}\right)$, drained shear stress $\left(G\right.$ '), the diffusion coefficient $\left(D_{p}\right)$ and the pore size (к). For nanoindentation process on soft samples such as the cells, probes with spherical tip geometry have been commonly employed to yield an accurate force-distance curve and meet the deformation criteria without causing plastic deformation the cells. Hence, the Hertzian model is commonly employed on the retrace curve of the force distance curve and the model is as follows,

$$
F=\left(\frac{8}{3}\right)\left(\frac{E}{1-\vartheta_{u}}\right) R^{\frac{1}{2}} \delta^{\frac{3}{2}} \ldots
$$

Where, $\mathrm{F}$ is the force on the tip, $\mathrm{E}$ is the Young's modulus, $\vartheta_{u}$ is undrained the Poisson's ratio, $\mathrm{R}$ is the radius of the indenter and $\delta$ is the indentation. By fitting the retrace curve to yield maximum linearity coefficient value, E can be obtained. Further, elastic modulus and shear modulus (G) are linearly related through the Poisson's ratio.

$$
E=2 G\left(1+\vartheta_{u}\right) \ldots(2)
$$

In the force-relaxation experiment, the cantilever is approached at high forward velocity due to which the cytoplasmic interstitial fluid does not have sufficient time to drain out of the compressed region. This is identified as the undrained scenario for poroelastic material [70,72]. The equation (1) becomes,

$$
F(0)=\left(\frac{16}{3}\right) G^{\prime} R^{\frac{1}{2}} \delta^{\frac{3}{2}} \ldots
$$


As the force reaches a plateau, the interstitial fluid redistributes in the cell and the force inflicted by the indenter is balanced by the stress in the elastic porous matrix only. This condition is termed as the drained condition and equation (1) becomes,

$$
F(\infty)=\left(\frac{8}{3}\right)\left(\frac{1}{1-\vartheta_{d}}\right) G^{\prime \prime} R^{\frac{1}{2}} \delta^{\frac{3}{2}}
$$

Where, $\vartheta_{d}$ is the drained Poisson's ratio.

Comparing equations 3 and 4 yields,

$$
\left(\frac{F(0)}{F(\infty)}\right)=2\left(1-\vartheta_{d}\right) \ldots(5)
$$

F (0) and F $(\infty)$ can be extracted from the force-relaxation curve. Equation 5 yields drained Poisson's ratio. By substituting $\vartheta_{d}$ in equation 4, we can obtain drained shear stress (G'). Moreover, equations 3 yields undrained shear stress $\left(G^{\prime}\right)$ as $F(0), R$ and $\vartheta_{d}$ ) are known quantities. There fails to exist any closed form analytical solution for indentation of a poroelastic infinite halfspace by a spherical indenter exists. However, an approximate solution is obtained by finiteelement simulation [73] given by,

$$
\frac{F(t)-F(\infty)}{F(0)-F(\infty)}=0.491 e^{-0.908 \sqrt{\tau}}+0.509 e^{-1.679 \tau} \ldots(6)
$$

Where, $\tau$ is the characteristic poroelastic time required for the force to relax. $F(t), F(0)$ and $F(\infty)$ are all extracted from the force relaxation curve. The diffusion constant $\left(D_{p}\right)$ of the porous membrane is related to the characteristic time by;

$$
\tau=\left(\frac{D_{p} t}{R \delta}\right) \ldots(7)
$$

Diffusion coefficient is further directly linked to the pore size given by,

$$
D_{p}=\frac{2 G \prime \prime\left(1-\vartheta_{d}\right)}{1-2 \vartheta_{d}} \kappa
$$

Where, $\kappa$ is the pore size. Equations 4, 5, 7 and 8 cumulatively yield the poroelasticity parameters such as G', $v_{d}, D_{p}$ and $\kappa$, respectively. 


\section{Data analysis of AFM experiments}

Bruker's Nanoscope analysis v1.9 was used to extract values corresponding to the F-R curves, which were then, analyzed using a custom-built MATLAB programming to yield values corresponding to various poroelastic parameters. Origin Pro Lab software was then used for statistical analysis and graphical presentation. After ensuring that these datasets satisfy normality criteria, One Way ANNOVA was performed to calculate the significance between the datasets.

\section{Supporting Information}

Supporting information comprises of five figures that support the main findings mentioned in the manuscript. These figures comprise of representative force-relaxation curves for Panc1, AsPC-1 and HUVECs under no treatment. Baseline experiments exhibiting temporal alterations in poroelasticity parameters $S$ phase Panc1 cells in the presence of the AFM tip applied stimulus, alterations in synchronous and asynchronous AsPC-1 cells in the presence of the AFM tip applied stimulus, comparative analysis of poroelasticity parameters in synchronous Panc1 and HUVECs without any treatment and lastly, alterations in drained shear stress during receptor dependent and independent endocytosis process.

\section{Declarations}

\section{Availability of data and materials}

The datasets used and/or analyzed during the current study are available from the corresponding author on reasonable request.

\section{Conflicts of Interest}

The authors declare that they haveno competing interests

\section{Funding}


This work is supported partly by National Institutes of Health grants CA78383, CA150190, NHLBI (\#HL140411) and Florida Department of Health (Cancer Research Chair Fund, Florida \#3J) to DM and Mayo Clinic Pancreatic Cancer SPORE Career Enhancement Award, Eagles fifth District Cancer Telethon-Cancer Research Fund and Jay and Deanie Stein Career Development Award for Cancer Research at Mayo Clinic Jacksonville, 2019 Benefactor Funded Champions for Hope Pancreatic Cancer to SB.

\section{Author Contributions}

T.A.K.- Data curation, formal analysis, investigation, methodology, original draft.

S.B.- Conceptualization, methodology, writing - review and editing, funding acquisition

D.M.- Conceptualization, funding acquisition.

\section{Acknowledgments}

Graphical abstract and Figure 7A schematic was created with Biorender.com.

\section{References}

1. Singh R, Lillard Jr JW: Nanoparticle-based targeted drug delivery. Experimental and molecular pathology 2009, 86:215-223.

2. Paciotti GF, Kingston DG, Tamarkin L: Colloidal gold nanoparticles: a novel nanoparticle platform for developing multifunctional tumor-targeted drug delivery vectors. Drug development research 2006, 67:47-54.

3. Wu L, Xie W, Zan H-M, Liu Z, Wang G, Wang Y, Liu W, Dong W: Platelet membrane-coated nanoparticles for targeted drug delivery and local chemo-photothermal therapy of orthotopic hepatocellular carcinoma. Journal of Materials Chemistry B 2020, 8:4648-4659.

4. Kulkarni T, Mukhopadhyay D, Bhattacharya S: Nanomechanical Insight of Pancreatic Cancer Cell Membrane during Receptor Mediated Endocytosis of Targeted Gold Nanoparticles. ACS Applied Bio Materials 2020, 4:984-994.

5. Mulens-Arias V, Balfourier A, Nicolás-Boluda A, Carn F, Gazeau F: Endocytosis-driven gold nanoparticle fractal rearrangement in cells and its influence on photothermal conversion. Nanoscale 2020, 12:21832-21849. 
6. He Z, Liu K, Scally L, Manaloto E, Gunes S, Ng SW, Maher M, Tiwari B, Byrne HJ, Bourke P: Cold atmospheric plasma stimulates clathrin-dependent endocytosis to repair oxidised membrane and enhance uptake of nanomaterial in glioblastoma multiforme cells. Scientific reports 2020, 10:1-12.

7. Enea M, Pereira E, Costa J, Soares ME, da Silva DD, de Lourdes Bastos M, Carmo HF: Cellular uptake and toxicity of gold nanoparticles on two distinct hepatic cell models. Toxicology in Vitro 2021, 70:105046.

8. Hoffman BD, Grashoff C, Schwartz MA: Dynamic molecular processes mediate cellular mechanotransduction. Nature 2011, 475:316-323.

9. Wernig F, Xu Q: Mechanical stress-induced apoptosis in the cardiovascular system. Progress in biophysics and molecular biology 2002, 78:105-137.

10. Zhou G, Zhang B, Tang G, Yu X-F, Galluzzi M: Cells nanomechanics by atomic force microscopy: focus on interactions at nanoscale. Advances in Physics: $X$ 2021, 6:1866668.

11. Jokhadar ŠZ, Iturri J, Toca-Herrera JL, Derganc J: Cell stiffness under small and large deformations measured by optical tweezers and atomic force microscopy: effects of actin disruptors CK-869 and jasplakinolide. Journal of Physics D: Applied Physics 2021, 54:124001.

12. Li M, Xi N, Wang Y-c, Liu L-q: Atomic force microscopy for revealing micro/nanoscale mechanics in tumor metastasis: from single cells to microenvironmental cues. Acta Pharmacologica Sinica 2021, 42:323-339.

13. Liao Y-X, Zhang Z-P, Zhao J, Liu J-P: Effects of fibronectin 1 on cell proliferation, senescence and apoptosis of human glioma cells through the PI3K/AKT signaling pathway. Cellular Physiology and Biochemistry 2018, 48:1382-1396.

14. Sanchez-Esteban J, Wang Y, Gruppuso PA, Rubin LP: Mechanical stretch induces fetal type II cell differentiation via an epidermal growth factor receptor-extracellular-regulated protein kinase signaling pathway. American journal of respiratory cell and molecular biology 2004, 30:76-83.

15. Wojcikiewicz EP, Zhang X, Moy VT: Force and compliance measurements on living cells using atomic force microscopy (AFM). Biological procedures online 2004, 6:1-9.

16. Guo X, Bonin K, Scarpinato K, Guthold M: The effect of neighboring cells on the stiffness of cancerous and non-cancerous human mammary epithelial cells. New Journal of Physics 2014, 16:105002.

17. Sun $T$, Zhang YS, Pang B, Hyun DC, Yang M, Xia Y: Engineered nanoparticles for drug delivery in cancer therapy. Angewandte Chemie International Edition 2014, 53:12320-12364.

18. Aschenbrenner L, Naccache SN, Hasson T: Uncoated endocytic vesicles require the unconventional myosin, Myo6, for rapid transport through actin barriers. Molecular biology of the cell 2004, 15:2253-2263.

19. Mofrad MR: Rheology of the cytoskeleton. Annual Review of Fluid Mechanics 2009, 41:433-453.

20. Ingber DE, Wang N, Stamenovic D: Tensegrity, cellular biophysics, and the mechanics of living systems. Rep Prog Phys 2014, 77:046603.

21. Hoffman BD, Crocker JC: Cell mechanics: dissecting the physical responses of cells to force. Annu Rev Biomed Eng 2009, 11:259-288.

22. Sneddon IN: The relation between load and penetration in the axisymmetric Boussinesq problem for a punch of arbitrary profile. International journal of engineering science 1965, 3:4757.

23. Hertz $\mathrm{H}$ : On the contact of rigid elastic solids and on hardness, chapter 6: Assorted papers by $\mathrm{H}$. Hertz. MacMillan, New York; 1882.

24. Packham D: Work of adhesion: contact angles and contact mechanics. International journal of adhesion and adhesives 1996, 16:121-128. 
25. Barthel E: Adhesive elastic contacts: JKR and more. Journal of Physics D: Applied Physics 2008, 41:163001.

26. Derjaguin BV, Muller VM, Toporov YP: Effect of contact deformations on the adhesion of particles. Journal of Colloid and interface science 1975, 53:314-326.

27. Fabry B, Maksym GN, Butler JP, Glogauer M, Navajas D, Fredberg JJ: Scaling the microrheology of living cells. Phys Rev Lett 2001, 87:148102.

28. Fredberg JJ, Stamenovic D: On the imperfect elasticity of lung tissue. J Appl Physiol (1985) 1989, 67:2408-2419.

29. Hildebrandt J: Comparison of mathematical models for cat lung and viscoelastic balloon derived by Laplace transform methods from pressurevolume data. The Bulletin of mathematical biophysics 1969, 31:651-667.

30. Maksym GN, Fabry B, Butler JP, Navajas D, Tschumperlin DJ, Laporte JD, Fredberg JJ: Mechanical properties of cultured human airway smooth muscle cells from 0.05 to $0.4 \mathrm{~Hz}$. Journal of Applied Physiology 2000, 89:1619-1632.

31. Mollaeian K, Liu Y, Bi S, Ren J: Atomic force microscopy study revealed velocity-dependence and nonlinearity of nanoscale poroelasticity of eukaryotic cells. Journal of the mechanical behavior of biomedical materials 2018, 78:65-73.

32. Bachrach NM, Valhmu WB, Stazzone E, Ratcliffe A, Lai WM, Mow VC: Changes in proteoglycan synthesis of chondrocytes in articular cartilage are associated with the time-dependent changes in their mechanical environment. Journal of biomechanics 1995, 28:1561-1569.

33. Guilak F, Mow VC: The mechanical environment of the chondrocyte: a biphasic finite element model of cell-matrix interactions in articular cartilage. Journal of biomechanics 2000, 33:16631673.

34. Moeendarbary E, Valon L, Fritzsche M, Harris AR, Moulding DA, Thrasher AJ, Stride E, Mahadevan $\mathrm{L}$, Charras GT: The cytoplasm of living cells behaves as a poroelastic material. Nature materials 2013, 12:253-261.

35. Jauvert S, Peyroux R, Richefeu V: A mechanical model for cell motility and tissue morphogenesis. Computer Methods in Biomechanics and Biomedical Engineering 2013, 16:13-14.

36. He DJ, Hu YH: A nonlinear visco-poroelasticity model for transversely isotropic gels. Meccanica 2020.

37. Florea C, Tanska P, Mononen ME, Qu CJ, Lammi MJ, Laasanen MS, Korhonen RK: A combined experimental atomic force microscopy-based nanoindentation and computational modeling approach to unravel the key contributors to the time-dependent mechanical behavior of single cells. Biomechanics and Modeling in Mechanobiology 2017, 16:297-311.

38. Bonfanti A, Fouchard J, Khalilgharibi N, Charras G, Kabla A: A unified rheological model for cells and cellularised materials. Royal Society Open Science 2020, 7.

39. Zhu X, Cirovic S, Shaheen A, Xu W: Investigation of fullerenol-induced changes in poroelasticity of human hepatocellular carcinoma by AFM-based creep tests. Biomech Model Mechanobiol 2018, 17:665-674.

40. Mollaeian K, Liu Y, Ren J: Investigation of nanoscale poroelasticity of eukaryotic cells using Atomic Force Microscopy. In ASME 2017 Dynamic Systems and Control Conference. American Society of Mechanical Engineers Digital Collection; 2017

41. Kulkarni T, Mukhopadhyay D, Bhattacharya S: Nanomechanical Insight of Pancreatic Cancer Cell Membrane during Receptor Mediated Endocytosis of Targeted Gold Nanoparticles. ACS Applied Bio Materials 2020.

42. Kulkarni T, Tam A, Mukhopadhyay D, Bhattacharya S: AFM study: Cell cycle and probe geometry influences nanomechanical characterization of Panc1 cells. Biochimica et Biophysica Acta (BBA)General Subjects 2019, 1863:802-812. 
43. Herrmann H, Bar H, Kreplak L, Strelkov SV, Aebi U: Intermediate filaments: from cell architecture to nanomechanics. Nat Rev Mol Cell Biol 2007, 8:562-573.

44. Mahaffy RE, Shih CK, MacKintosh FC, Kas J: Scanning probe-based frequency-dependent microrheology of polymer gels and biological cells. Phys Rev Lett 2000, 85:880-883.

45. Bausch D, Thomas S, Mino-Kenudson M, Fernandez-del Castillo C, Bauer TW, Williams M, Warshaw AL, Thayer SP, Kelly KA: Plectin-1 as a Novel Biomarker for Pancreatic Cancer. Clinical Cancer Research 2011, 17:302-309.

46. $\mathrm{Xu} \mathrm{P}, \mathrm{Liu} \mathrm{H}$ : Models of microfibril elastic modulus parallel to the cell axis. Wood Science and Technology 2004, 38:363-374.

47. Lowery J, Kuczmarski ER, Herrmann H, Goldman RD: Intermediate Filaments Play a Pivotal Role in Regulating Cell Architecture and Function. J Biol Chem 2015, 290:17145-17153.

48. Kim S, Coulombe PA: Intermediate filament scaffolds fulfill mechanical, organizational, and signaling functions in the cytoplasm. Genes Dev 2007, 21:1581-1597.

49. Sanghvi-Shah R, Weber GF: Intermediate filaments at the junction of mechanotransduction, migration, and development. Frontiers in cell and developmental biology 2017, 5:81.

50. Ingber DE: Tensegrity-based mechanosensing from macro to micro. Progress in biophysics and molecular biology 2008, 97:163-179.

51. Shin W, Ge L, Arpino G, Villarreal SA, Hamid E, Liu H, Zhao WD, Wen PJ, Chiang HC, Wu LG: Visualization of Membrane Pore in Live Cells Reveals a Dynamic-Pore Theory Governing Fusion and Endocytosis. Cell 2018, 173:934-945 e912.

52. Wu LG, Hamid E, Shin W, Chiang HC: Exocytosis and endocytosis: modes, functions, and coupling mechanisms. Annu Rev Physiol 2014, 76:301-331.

53. Zhang X, Ren J, Wang J, Li S, Zou Q, Gao N: Receptor-mediated endocytosis generates nanomechanical force reflective of ligand identity and cellular property. Journal of cellular physiology 2018, 233:5908-5919.

54. Chim YH, Mason LM, Rath N, Olson MF, Tassieri M, Yin H: A one-step procedure to probe the viscoelastic properties of cells by Atomic Force Microscopy. Scientific reports 2018, 8:1-12.

55. Robinson MS, Watts C, Zerial M: Membrane dynamics in endocytosis. Cell 1996, 84:13-21.

56. Zhang X, Ren J, Wang JR, Li SX, Zou QZ, Gao N: Receptor-mediated endocytosis generates nanomechanical force reflective of ligand identity and cellular property. Journal of Cellular Physiology 2018, 233:5908-5919.

57. Staszewska I, Fischer I, Wiche G: Plectin isoform 1-dependent nuclear docking of desmin networks affects myonuclear architecture and expression of mechanotransducers. Human Molecular Genetics 2015, 24:7373-7389.

58. Staunton JR, Fuhrmann A, Nandakumar V, Banyai N, Davies PCW, Ros R: AFM Stiffness Nanotomography of Normal, Metaplastic and Dysplastic Human Esophageal Cells. Biophysical Journal 2011, 100:190-190.

59. Li QS, Lee GYH, Ong CN, Lim CT: AFM indentation study of breast cancer cells. Biochemical and Biophysical Research Communications 2008, 374:609-613.

60. Puech PH, Poole K, Knebel D, Muller DJ: A new technical approach to quantify cell-cell adhesion forces by AFM. Ultramicroscopy 2006, 106:637-644.

61. Sen S, Subramanian S, Discher DE: Indentation and adhesive probing of a cell membrane with AFM: Theoretical model and experiments. Biophysical Journal 2005, 89:3203-3213.

62. Wei F, Lan F, Liu B, Liu LQ, Li GY: Poroelasticity of cell nuclei revealed through atomic force microscopy characterization. Applied Physics Letters 2016, 109.

63. Liu Y, Mollaeian K, Ren J: Finite element modeling of living cells for AFM indentation-based biomechanical characterization. Micron 2019, 116:108-115. 
64. Kang T, Park C, Lee B-J: Investigation of biomimetic shear stress on cellular uptake and mechanism of polystyrene nanoparticles in various cancer cell lines. Archives of pharmacal research 2016, 39:1663-1670.

65. Kang T, Cho Y, Park C, Kim S-D, Oh E, Cui J-H, Cao Q-R, Lee B-J: Effect of biomimetic shear stress on intracellular uptake and cell-killing efficiency of doxorubicin in a free and liposomal formulation. International journal of pharmaceutics 2016, 510:42-47.

66. Sivaramakrishnan S, Schneider JL, Sitikov A, Goldman RD, Ridge KM: Shear stress induced reorganization of the keratin intermediate filament network requires phosphorylation by protein kinase C 3 . Molecular biology of the cell 2009, 20:2755-2765.

67. Rodino-Janeiro BK, Gonzalez-Peteiro M, Ucieda-Somoza R, Gonzalez-Juanatey JR, Alvarez E: Glycated albumin, a precursor of advanced glycation end-products, up-regulates NADPH oxidase and enhances oxidative stress in human endothelial cells: molecular correlate of diabetic vasculopathy. Diabetes-Metabolism Research and Reviews 2010, 26:550-558.

68. Pal K, Al-Suraih F, Gonzalez-Rodriguez R, Dutta SK, Wang E, Kwak HS, Caulfield TR, Coffer JL, Bhattacharya S: Multifaceted peptide assisted one-pot synthesis of gold nanoparticles for plectin-1 targeted gemcitabine delivery in pancreatic cancer. Nanoscale 2017, 9:15622-15634.

69. Kulkarni T, Angom RS, Das P, Bhattacharya S, Mukhopadhyay D: Nanomechanical insights: Amyloid beta oligomer-induced senescent brain endothelial cells. Biochimica et Biophysica Acta (BBA)-Biomembranes 2019, 1861:183061.

70. Wang HF: Theory of linear poroelasticity with applications to geomechanics and hydrogeology. Princeton university press; 2017.

71. Selvadurai A: Stationary damage modelling of poroelastic contact. International Journal of Solids and Structures 2004, 41:2043-2064.

72. Detournay E, Cheng AH-D: Fundamentals of poroelasticity. In Analysis and design methods. Elsevier; 1993: 113-171

73. Hu Y, Zhao X, Vlassak JJ, Suo Z: Using indentation to characterize the poroelasticity of gels. Applied Physics Letters 2010, 96:121904. 


\section{Supplementary Files}

This is a list of supplementary files associated with this preprint. Click to download.

- SupplementaryInformation.docx 